\title{
PENSER LA PLURIACTIVITÉ DANS LE HAUT-LANGUEDOC : REGISTRES DE JUSTIFICATION ET DISPOSITIF D'ACCOMPAGNEMENT
}

Hélène Tallon, Michel Dulcire, Aurélie Dubien

Armand Colin | «Revue d’Économie Régionale \& Urbaine »

2013/1 février | pages 93 à 117

ISSN 0180-7307

ISBN 9782200928674

Article disponible en ligne à l'adresse :

http://www.cairn.info/revue-d-economie-regionale-et-urbaine-2013-1-page-93.htm

\section{Pour citer cet article :}

Hélène Tallon et al., « Penser la pluriactivité dans le Haut-Languedoc : registres de justification et dispositif d'accompagnement », Revue d'Économie Régionale \& Urbaine 2013/1 (février), p. 93-117.

DOI 10.3917/reru.131.0093

Distribution électronique Cairn.info pour Armand Colin.

(C) Armand Colin. Tous droits réservés pour tous pays.

La reproduction ou représentation de cet article, notamment par photocopie, n'est autorisée que dans les limites des conditions générales d'utilisation du site ou, le cas échéant, des conditions générales de la licence souscrite par votre établissement. Toute autre reproduction ou représentation, en tout ou partie, sous quelque forme et de quelque manière que ce soit, est interdite sauf accord préalable et écrit de l'éditeur, en dehors des cas prévus par la législation en vigueur en France. Il est précisé que son stockage dans une base de données est également interdit. 


\title{
Penser la pluriactivité dans le Haut-Languedoc : registres de justification et dispositif d'accompagnement
}

\section{Thinking about the pluriactivity in Haut-Languedoc: registers of justification and apparatus of accompaniment}

\author{
Hélène TALLON* \\ CIRAD-ES (UMR Tétis) \\ htallon@gmail.com
}

\author{
Michel DulCIRE \\ CIRAD-ES (UMR Innovation) \\ michel.dulcire@cirad.fr

\section{Aurélie Dubien} \\ AgroSup Dijon \\ aurelie.dubien@gmail.com
}

Mots clef : accompagnement, justification, pluriactivité, territoire

Keywords : accompaniment, justification, pluriactivity, rural area

Classification JEL : R11 


\section{Résumé}

Cet article interroge les formes de légitimation de la pluriactivité sur lesquelles s'appuient les organismes de soutien aux projets d'activité d'un territoire. En Languedoc-Roussillon, de nombreux organismes de développement territorial et d'accompagnement s'intéressent à la pluriactivité, avec des visions parfois opposées sur ses impacts dans l'économie d'un territoire. Pour comprendre ces divergences, nous avons relevé les principaux registres de justification rencontrés en Languedoc-Roussillon, et dans un espace rural plus restreint, le Haut-Languedoc. Nous constatons que ces registres differrent de ceux énoncés par les pluriactifs eux-mêmes, et sont centrés autour de la capacité de résilience de la pluriactivité, véritable atout dans la gestion des crises mais qui la confine dans la dimension du "petit", associée à l'idée de "fragile». Loin d'un "monde » cohérent, des visions politiques du travail divergentes s'expriment, avec lesquelles le dispositif d'accompagnement à la pluriactivité doit faire des compromis.

\section{Abstract}

How do rural activities support organizations justify the pluriactivity in their policies? The pluriactivity is becoming an important issue in Languedoc-Roussillon. Nevertheless the resources of 'accompaniment' do not always share the same views concerning the pluriactivity contribution in activity development and dynamic. They bring into sharp relief the tensions and ambiguities in public policy. In order to understand the divergences observed, we analyzed the forms of justification on which the discourses are built. Our results show that the way pluriactivity contribute to activities' resilience is considered as a real asset in crisis management. However it limits the pluriactivity to the dimension of the "small" and "fragile». Moreover the approach of pluriactivity remains different between organisations and pluriactives. Political views of work confront one another, contrasting with the ideal of a coherent "world». Nonetheless the apparatus of accompaniment should take this situation into account.

\section{-1 - Introduction}

Le droit du travail définit la pluriactivité comme « l'exercice simultané ou successif par une même personne de plusieurs activités professionnelles différentes " (CASAUX, 1993). Au-delà de cette définition, nous considérons par ailleurs qu'il y a situation de pluriactivité lorsque la même activité est exercée sous plusieurs statuts sociaux différents (salariat en particulier), car les statuts régissent les cadres réglementaires des pratiques et ont de fait une incidence essentielle sur les stratégies de combinaison d'activités. En ce qui concerne les activités agricoles, notre définition ne fait pas de distinction entre double activité, agriculture à temps partiel, multiactivité..., et intègre une partie de ce qui est considéré par les organismes professionnels agricoles comme de la diversification, soit les activités non liées à la production mais exercées sous statut agricole (vente et transformation, camping sur la ferme). Cette définition très large, dont les termes sont en eux-mêmes objet de débat (FOUQUET, 1998), nous permet de rendre compte de la palette de représentations de la pluriactivité que nous trouvons sur un territoire donné.

Après un demi-siècle de marginalisation, la pluriactivité refait parler d'elle et progresse nettement dans l'emploi salarié, où elle représente en 2005 5\% de cet emploi, avec une progression de l'ordre de 15\% entre 2003 et 2005 (BEFF, 2006). Sa progression est plus discrète dans l'emploi indépendant, mais elle y est en proportion beaucoup plus importante : elle concernerait entre 10 à $26 \%$ des actifs 
(BOUDY, 2009). Il est important de noter que cette progression se retrouve dans tous les pays occidentaux, et cela quelles que soient les formes de soutien ou de limites qui lui sont appliquées (BOUDY, 2009). Les statistiques concernant la pluriactivité sont très variables selon les bases et les sources disponibles, et sont difficiles à manier. Mais au-delà des données brutes, ces statistiques sont utiles pour identifier des tendances. La pluriactivité est une forme d'activité structurelle des territoires ruraux (MAYAUD, 1988 ; MENDRAS, 1976), et sa référence historique à l'activité paysanne fait parfois oublier qu'elle concerne tous les domaines d'activité, depuis tous temps. D'après les données fiscales citées par BouDy (2009), 2/3 des non salariés pluriactifs sont artisans, commerçants ou appartiennent à des professions libérales. Cependant la pluriactivité est loin d'être reconnue comme une forme d'activité aussi enviable que l'emploi unique. Dans nos sociétés où le système de protection sociale a été pensé à partir du modèle de l'emploi salarié exercé à plein temps et tout au long de la vie, la monoactivité est la norme : s'en écarter peut être difficile sur le plan juridique, fiscal ou identitaire. Les collectivités locales en charge de l'activité économique sont ainsi peu à l'aise avec la pluriactivité, et le spectre de la précarité et du maintien de populations dans des formes de travail disqualifiées les incitent à la prudence. Par conséquent leurs politiques concernant la pluriactivité sont ambigües (COUTURIER, 1994 ; TALLON, 2011b) et très diversement mises en œuvre. Ces collectivités ont pourtant à composer avec des espaces ruraux en mutation, pour certains véritables espaces d'expérimentation de nouvelles formes de travail et d'emploi. En témoigne le développement de formes d'activités dites atypiques, informelles, alternatives, etc. parmi lesquelles figurent de nombreuses expériences de combinaisons d'activités professionnelles, qui induisent un rapport spécifique au territoire, davantage cadre de vie, d'activité, et d'épanouissement du projet personnel, que comme lieu producteur de ressource identifiée et évaluée pour une activité économique (GUMUCHIAN et PECQUEUR, 2007). Les travaux récents concernant la dynamique des espaces ruraux montrent l'intérêt de repérer les jeux sociaux qui animent les pratiques professionnelles singulières d'un espace donné, et qui œuvrent à l'émergence et à la mobilisation de compétences spécifiques (BEL, 2009). Cette approche revient à confronter le regard porté par les politiques publiques sur les évolutions territoriales avec les jeux d'acteurs, les trajectoires d'évolution des activités productives et sociales, les mobilités et les relations entre les espaces. C'est dans ce sens que la pluriactivité fait bouger les lignes des dispositifs d'accompagnement, en invitant à repenser tant les formes de l'activité professionnelle que ses finalités. Dans ce mouvement de renaissance de la pluriactivité, la compréhension des motivations sous-jacentes à son exercice, et en négatif de celles présidant à son soutien, se pose donc avec acuité.

L'analyse que nous faisons dans cet article de la prise en compte de la pluriactivité par les organismes de soutien à la création d'activité repose sur une approche axée sur la justification, telle que développée par BOLTANSKI et THÉVENOT (1991) ; justification de la prise en compte, ou de l'absence de prise en compte, de la pluriactivité par ces différentes structures. Le choix de s'appuyer sur le concept de justification, permet, via la sociologie des régimes d'action, de situer l'action, c'est-à-dire de l'appréhender dans sa "dynamique d'ajustement » qui prend en compte les objets, les institutions, les individus mais aussi les contraintes extérieures autour desquelles les personnes 
ajustent leurs actions (CORCUFF, 1998). La justification implique un engagement de la part d'une institution ou d'un individu dans une action collective soumise au jugement et à la critique, qui sous-entend une incertitude ou un conflit d'évaluation (THEVENOT, 1996). C'est ainsi l'action, lorsqu'elle peut se dérouler, qui fournira la preuve de la justesse de l'argumentation. L'action valide la justification. De la riche analyse du jugement en situation et des " mondes » de BOLTANSKI et THÉVENOT (1991), retenons que, dans la pluralité des régimes de justification, les équilibres sont en réajustement permanent, et que certaines formes de confrontation sont résolues par le compromis. Celui-ci sera donc le moyen de dépasser les tensions entre les registres de justification et permettra leur compatibilité locale. Les structures portant un projet de territoire se doivent d'argumenter leurs choix de développement et les actions qui s'y rapportent, notamment dans leurs documents de communication (charte de développement, contrat de Pays, contrat de projet État-Région...), en se basant entre autres sur les caractéristiques de leur territoire d'action et sur des enjeux de développement identifiés. L'orientation donnée aux actions menées par ces structures de développement territorial nous permet d'identifier les visions qu'elles ont de l'activité du territoire. Les structures d'accompagnement auront, quant à elles, plutôt tendance à baser leurs orientations sur les besoins des publics qu'elles prennent en charge. Cependant, des liens doivent exister entre les objectifs d'une structure d'accompagnement et la politique institutionnelle à laquelle elle fera appel pour réaliser ses diverses actions. Ces structures, par l'intermédiaire de conventions de partenariat, sont tenues, pour bénéficier des financements qui leur sont alloués, de se conformer aux obligations énoncées par les politiques régionales et nationales en matière de durée d'accompagnement d'une personne, de réalisation d'objectifs chiffrés, etc. De même, un projet porté par une collectivité territoriale ne peut être déconnecté de ses divers échelons financiers et opérationnels. De ce fait, le concept de justification apparaît comme central dans la mise en place des actions, et fait partie intégrante des stratégies de développement des structures de développement territorial comme des structures d'accompagnement. Le principe de l'accord qui fonde les possibilités de coopération et d'adhésion aux actions des collectivités comme des individus ne peut en effet exister sans rapport à un principe supérieur commun (BOLTANSKI et THEVENOT, 2009).

A l'échelle de nos deux territoires, la Région Languedoc-Roussillon et le HautLanguedoc, l'accompagnement à la pluriactivité se réfere-t-il à un principe commun ? Ou, à l'inverse, peut-on trouver dans les éventuels décalages d'objectifs et d'enjeux assignés à la pluriactivité une explication aux lacunes actuelles de l'accompagnement à la pluriactivité ? 


\section{- 2 - \\ Quel accompagnement pour quels enjeux ?}

\subsection{Les bases du soutien à la pluriactivité}

En France, les rôles alloués à la pluriactivité et les justifications sur lesquelles s'appuient les dispositifs de soutien à la pluriactivité diffèrent : forme d'adaptation aux crises sectorielles ou aux mutations de l'emploi ; pérennisation des activités ou maintien des populations ; gestion de la précarité ; développement des secteurs économiques ; etc. Quant aux pluriactifs, enjeux et motivations sont imbriqués dans les parcours de vie. Nous distinguons trois catégories d'enjeux : un enjeu économique ; un enjeu territorial ; un enjeu identitaire et social.

L'enjeu économique est de loin le plus analysé. La pluriactivité permet de pallier la faible rémunération de la première activité par l'apport d'un revenu complémentaire, et peut aussi avoir pour rôle d'assurer les investissements nécessaires (BUTAULT et al., 1999). Elle augmente la rentabilité interne des activités lorsque les outils de productions, les charges et la clientèle sont mutualisés. Elle alimente alors la résistance aux aléas économiques et climatiques par la flexibilité interne du système, une activité pouvant être développée ou mise en veille selon les besoins (ROBERTSON et al., 2008). La pluriactivité est globalement plus rémunératrice que la monoactivité : en 1990 moins de 9,6\% des personnes déclarant deux régimes fiscaux différents avaient un revenu annuel inférieur à $50000 \mathrm{~F}$ contre 14,2\% chez les commerçantsartisans, $18,2 \%$ chez les professions libérales, 19,7\% chez les salariés et 48,4\% chez les agriculteurs (BICHE et al., 1996). Notons que chez les agriculteurs, seuls les ménages pluriactifs atteignent un niveau de vie comparable au revenu moyen des autres individus (BuTAULT et al.,1999). Cependant, des analyses plus fines montrent que ce rapport gagnant/gagnant n'est pas si évident, et la pluriactivité a été largement combattue en France dès les années 1960 par les syndicats professionnels agricoles qui l'accusaient de scléroser la dynamique de modernisation des structures et de la filière. La politique européenne agricole est plutôt favorable à l'heure actuelle à la pluriactivité, dans la mesure où la politique de développement rural menée depuis la fin des années 1980 fait de la diversification et du renforcement des liens entre l'agriculture et les autres secteurs de l'économie un moyen d'adaptation du secteur agricole aux évolutions économiques défavorables aux territoires non compétitifs. Mais la controverse autour de la prise de position de Mariann FisCHER BOEL, commissaire européen à l'agriculture, qui déclarait en 2006 au Financial Times que la progression de l'agriculture à temps partiel serait visible dans les prochaines années puisque les agriculteurs devront compenser la baisse annoncée du soutien européen à l'agriculture par des revenus extérieurs (FISCHER BOEL, 2006), montre que la pluriactivité n'est plus acceptée lorsque qu'elle met en exergue la fragilité de tout un secteur économique et l'identité d'une profession. "Mariann FISCHER BOEL voudrait une sous-rémunération chronique des paysans " [Régis HOCHART, porteparole de la Confédération paysanne (HOCHART, 2007)], "vos propos ont alors pour objectif de préparer la classe paysanne européenne à endosser l'habit des 'intermittents de l'agriculture' " [extrait de la lettre ouverte à Mariann FISCHER BOEL, par Pierre PAGESSE, président du MOMAGRI, le Mouvement pour une Organisation Mondiale 
de L'AGRIculture (PAGESSE, 2006)], " oui à la pluriactivité choisie, non à la pluriactivité subie! » [discours de Jean-Michel LEMÉTAYER, Président de la Fédération Nationale des Syndicats d'Exploitants Agricoles, en réponse aux propos de Mariann FisCHER BOEL (LEMÉTAYER, 2007)]. Le débat est passionné et complexe, au sein d'une Europe où les taux de pluriactivité agricole varient de $60 \%$ pour la Suède à moins de $20 \%$ pour la Belgique, avec une moyenne de 27\% pour l'Europe à 25 (EUROSTAT, 2003, cité par BouDY, 2009) et où les politiques nationales sont hétérogènes en la matière.

De plus, pluriactivité et précarité sont bien souvent deux termes associés. Certaines formes de pluriactivité se développent à partir d'activités réduites, peu rémunératrices et touchant des personnes particulièrement exposées aux conditions inégalitaires d'accès à l'emploi. La pluriactivité salariée concerne principalement les femmes : en France elles représentent $85 \%$ des salariés qui exercent leur profession pour plusieurs employeurs (BEFFY, 2006). Elles compensent par ce biais une durée du travail et un salaire insuffisants. L'emploi à temps partiel en forte progression (soit 18\% des emplois au début des années 2000 contre 8\% au début des années 1970) représente $31 \%$ des emplois féminins pour 5\% des emplois masculins (BELORGEY et al., 2000). Par ailleurs, le repeuplement des campagnes françaises observé ces 20 dernières années opère sur « un désir de campagne » (HERviEu et VIARD., 1996), mais il provient aussi plus récemment du développement de la précarité et du chômage dans les pôles urbains (PAGES, 2004). Ces exclus valorisent le mode de vie rural et relativisent ses contraintes et ses difficultés par l'intégration de représentations positives de son cadre de vie et de sa sociabilité. La faible offre d'emploi et les salaires bas des territoires ruraux (BLANC, 2003) pousse à l'adaptation aux handicaps de ces territoires par l'innovation mais favorise aussi l'économie informelle, très souvent pluriactive et par définition précarisante.

L'enjeu territorial est largement défendu, et semble être le plus consensuel. En effet, en intégrant la pluriactivité au pilier du développement rural, l'Union Européenne a entériné l'importance de l'agriculture dans la structure de l'emploi et de l'activité des territoires ruraux. La diminution drastique du nombre d'agriculteurs dans les territoires ruraux fragiles, suite à la politique de modernisation de l'agriculture des années 1960, a accentué l'effet d'abandon des territoires, déjà bien marqué par un siècle d'exode rural. La pluriactivité est devenue un élément des politiques nationales et locales d'aménagement du territoire, qui ne sont pas resté cantonnées au secteur agricole, mais qui se sont étendues à l'ensemble des activités rurales. Permettre le développement d'activités réduites ou intermittentes, adaptées aux handicaps structurels de l'agriculture montagnarde et de manière plus générique aux marchés du travail spécifiques des territoires ruraux (salaires bas, potentialités de valorisation des diplômes réduites, spécialisation des activités) (BLANC, 2003), apparaît depuis une vingtaine d'années comme un moyen de garder des « campagnes vivantes » en attirant des populations actives qui vont maintenir les services et les infrastructures de base, et dans le même mouvement les liens sociaux. C'est aussi le moyen d'assurer l'expansion de secteurs économiques saisonniers, comme le tourisme en montagne ou en bordure littorale : à titre d'exemple, en période hivernale le massif de la Tarentaise (Savoie) compte 18400 emplois de plus par rapport au reste de l'année, emplois occupés par des saisonniers qui sont à 63\% 
pluriactifs (ASSEDIC des Alpes, 2008). Cette relation qui est faite entre zones de faible productivité agricole, saisonnalité et pluriactivité, devrait donc dessiner dans le paysage rural des territoires de pluriactivité. Mais là encore les espaces à fort taux de pluriactivité ne se trouvent pas forcément là où on les attend. Si la montagne se caractérise par un faible taux d'agriculteurs à temps complet, la pluriactivité n'y est guère plus développée qu'ailleurs, 21,4\% contre 20,4\% pour la moyenne nationale (Enquêtes structures Agreste 2003, cité par BouDY, 2009). C'est en zone périurbaine que la pluriactivité agricole est la plus développée, ce phénomène étant observable dans l'ensemble de l'Europe. Une étude réalisée en Midi-Pyrénées met en évidence l'importance de la pluriactivité en zone périurbaine ainsi que dans les communes sous influence d'un pôle rural (respectivement $23,1 \%$ et $25,6 \%$, contre $15,8 \%$ pour l'ensemble de la région Midi-Pyrénées), alors que la diversification (tourisme à la ferme et vente directe), associée à l'image des paysages ruraux, reste à distance des villes (TRIBOULET et al., 1999). Il n'y a donc pas de déterminisme territorial de la pluriactivité. Son développement est corrélé à de nombreux facteurs en interactions dynamiques. Il s'appuie certes sur la spécificité du contexte économique local : « La faiblesse des ressources non agricoles [...] traduit une géographie inégalitaire des opportunités locales et par extension des possibilités de pluriactivité. " (SIMON, 2002), mais aussi dans des imbrications subtiles et parfois complexes avec l'histoire et l'héritage du territoire, son organisation sociale et politique, son identité.

Deux éléments conditionnent cependant plus particulièrement le développement de la pluriactivité. Tout d'abord la présence d'emplois salariés dans le bassin d'emploi. La figure actuellement quelque peu dépassée du " travailleur hors l'usine » qui combine le salariat de l'usine avec une activité indépendante, agricole ou non, et plus ou moins affichée, est bien représentée dans les espaces ruraux industriels. Les horaires concentrés et décalés favorisent cette pluriactivité, la plage de temps libre l'après-midi lors du travail de nuit par exemple offrant la possibilité d'une deuxième activité. A cet emploi industriel aujourd'hui en déclin se substitue l'emploi dans les activités de service, en plein développement dans les territoires ruraux, et caractérisé par son temps partiel. La flexibilité des horaires, lorsqu'elle est maîtrisée, peut être un atout dans le développement d'une deuxième activité. Le deuxième élément concerne le cycle même de l'activité : qu'elle soit saisonnière ou intermittente, et la pluriactivité apparaît alors massivement. Le tourisme de montagne dessine nettement deux périodes d'activités, l'hiver et l'été, tandis que le tourisme littoral se concentre sur la période estivale. Le spectacle vivant alterne périodes d'activité intenses et périodes creuses, consacrées à la création, mais qui laissent des possibilités d'activités annexes (enseignement sous forme de stages, etc.). Ainsi 58\% des artistes intermittents du spectacle exercent au moins deux métiers du spectacle (BUREAU et al., 2009). Les revenus moyens dégagés par l'activité artistique incitent à la pluriactivité, souvent informelle du fait des règles d'intégration des droits à l'assurance chômage spécifique à ces professions : $50 \%$ des artistes de la musique et du chant gagnent un salaire annuel inférieur à $6643 €$ (contre plus de $18000 €$ pour des salariés ne travaillant pas du tout dans le spectacle vivant). Le recours à la pluriactivité dans les deux secteurs du tourisme et du spectacle est structurel. 
L'enjeu identitaire et social est lui aussi important à considérer pour comprendre les choix de pluriactivité. Dans la très grande majorité des cas, les migrations vers le rural (et en particulier vers le rural le plus reculé) correspondent, pour l'individu, à la recherche d'une meilleure qualité de vie (FONT, 2000) et à l'expression d'un projet de vie personnel ou familial. Ce renouveau de l'espace rural est porté par des " ressorts propres de développement" (MORA, 2008) face aux nouveaux défis de mondialisation et d'individualisation. Dans les nouvelles formes d'entrepreneuriat rural la pluriactivité est très développée, et on peut observer que le fonctionnement des systèmes d'activités pluriactifs répond plus à des logiques existentielles qu'économiques (SALEILLES, 2007). Il révèle une autre conception du travail, correspondant à une recherche de valorisation de savoirs non académiques, à la mobilisation de compétences acquises tout au long du parcours professionnel mais aussi dans la sphère privée, et dans laquelle est reconnue l'utilité sociale du travail réalisé (LAURENT et al., 2006). Dans la sphère des activités de création, et chez les écrivains qu'elle étudie en particulier, HEINICH (2008) considère la pluriactivité comme une norme et non pas comme une anomalie : dans la mesure où la création relève du régime « vocationnel » de l'activité, on ne travaille pas pour gagner sa vie mais on gagne sa vie pour exercer son activité. Les artistes font des compromis pour exister sur des marchés peu rémunérateurs. La pluriactivité chez les artistes est d'ailleurs plus qu'une nécessité économique. La pratique et la valorisation de la pluriactivité font partie de leur démarche professionnelle. Beaucoup d'activités annexes leur permettent d'actualiser certaines compétences et leur assurent une légitimité professionnelle par l'ouverture aux rencontres et aux expériences qu'elles procurent. À l'intérieur d'une multiplicité d'activités dont l'activité de référence n'est pas celle qui fait vivre, l'enjeu identitaire est clair : " pouvoir se dire à soi-même et à autrui, et pouvoir être dit par autrui » (BUREAU et al., 2009). Enfin, à mi-chemin entre construction identitaire et création de lien social se trouvent les activités de « bricole » (WEBER, 2009), activités réalisées en marge d'une activité professionnelle, salariée la plupart du temps, et dont les fonctions sont multiples : signe de la capacité de prendre en main une activité "à soi ", système de don qui permet de s'insérer et d'être reconnu dans la sphère sociale, valorisation de savoir-faire, ou encore transmission patrimoniale.

\subsection{Un accompagnement à construire dans la situation}

Les raisons qui poussent à la mise en œuvre d'une pluriactivité sont donc nombreuses et parfois complexes, la décision étant le fruit d'une adaptation constante et dynamique au contexte économique et territorial de l'activité mais aussi de négociations avec soi-même, ses motivations axiologiques et les équilibres sociaux et familiaux attendus du projet. Dans le cas d'un projet pluriactif, l'accompagnement doit dépasser les frontières sectorielles des activités professionnelles et les formes normées de la création d'activité, et les échanges territoriaux entre acteurs doivent se construire et se structurer vers des " modes de gouvernance territoriale " (SOL ET Civilisation, 2010). Dans ce contexte il n'est pas étonnant de constater, comme le confirmeront les résultats présentés dans ce texte, que si la pluriactivité commence à trouver sa place au sein des politiques de développement rural et d'accompagnement de projet, sa prise en compte effective est encore faible. Ainsi, de nombreux porteurs de projets dits « atypiques », interrogés en Bretagne, soulignent la frilosité des autorités 
territoriales dans l'appui qui leur est proposé, alors que les leviers d'action existent en marge des politiques de niveau supérieur (AUBREE et MARECHAL, 2008). Les finalités de l'accompagnement à la pluriactivité des organismes de soutien sont hétérogènes et découlent directement de visions distinctes des apports de la pluriactivité pour les individus, le territoire ou la profession. Elles conditionnent ainsi les modalités d'accompagnement, dont quatre ont pu être relevées en Rhône-Alpes (LAURENT et al., 2006). La première consiste à pérenniser les situations de pluriactivité en adaptant les conditions de vie et de travail des personnes concernées (par exemple par la réhabilitation de logements pour les travailleurs saisonniers, par la mise en place de formations adaptées comme la formation bi-qualifiante, par des assouplissements ciblés de la réglementation, etc.). La deuxième consiste à considérer la pluriactivité comme une situation transitoire et à encourager, voire accompagner un retour à la monoactivité quel que soit le statut final envisagé (installation progressive agricole via un recours temporaire à la pluriactivité permettant d'assurer un revenu au ménage le temps de la montée en puissance de l'exploitation, ou au contraire sortie progressive de l'activité indépendante vers le salariat). La troisième consiste à construire une situation administrative de monoactivité qui permette aux pluriactifs de bénéficier des droits liés à un statut social (salariat dans le cas de groupements d'employeurs, de coopératives d'activité, de portage salarial ou indépendance par la reconnaissance d'activités liées à l'exploitation - agrotourisme, artisanat... - et rattachées au statut d'activité agricole). Enfin la dernière consiste à travailler à la mise en place d'un statut de l'activité qui confère des droits et une protection sociale dans la durée et qui permette une reconnaissance sociale de la polyvalence et de la mobilité professionnelle des individus. On peut dès lors s'interroger sur la manière dont les dispositifs d'accompagnement, qui relayent les politiques territoriales ou qui en sont pour le moins tributaires par leurs financements, s'accommodent de prises en compte aussi divergentes de la pluriactivité.

\section{- 3 - \\ Une pluriactivité diversement justifiée en Languedoc-Roussillon}

Pour tenter de répondre à la question des justifications autour desquelles se retrouvent ou non les dispositifs d'accompagnement à la pluriactivité, nous nous appuyons sur les travaux de recherche du programme PSDR "INTERSAMA ». Ce programme, "Insertion Territoriale des Systèmes d'Activités des Ménages Agricoles ", mené en Languedoc-Roussillon, est un projet de recherche-action du programme Pour et Sur le Développement Régional (PSDR3), portant sur les problématiques de la pluriactivité et de son accompagnement dans les territoires ruraux. Ces travaux ont montré l'hétérogénéité des modes de prise en compte de la pluriactivité, qui différencient les structures d'accompagnement les unes des autres en la matière. Au coeur même de cette hétérogénéité, nous souhaitions observer la place effective occupée par la pluriactivité, dans les discours et les actions des organisations. Pour cela, une première analyse des documents de communication des structures d'accompagnement de la région Languedoc-Roussillon a été réalisée en 2009 
(Bernardini et Pérez Chueca, 2009). Un focus a été réalisé dans un deuxième temps sur un territoire plus restreint de l'espace régional, le Haut-Languedoc héraultais (DUBIEN, 2010). Dans cette seconde étape, les documents " projets» des structures de développement territorial et des structures d'accompagnement ont été passés au crible. Une trentaine, soit la quasi-totalité des organisations composant le dispositif d'accompagnement du Haut-Languedoc, ont ainsi été étudiées : les structures d'accompagnement proprement dites; les collectivités locales de rang supérieur comme la Région, le Département, le Pays, le Parc Naturel Régional ; et un échantillon de communautés de communes. Nous en avons dégagé les registres mobilisés pour justifier les actions (ou l'absence d'action) liées à la pluriactivité. Il faut noter que très peu de structures se réfèrent explicitement à la pluriactivité dans leurs documents écrits, même lorsqu'elle est significative dans les actions menées. Pour avoir néanmoins accès à l'information recherchée et la confronter aux données des documents écrits, ces analyses de contenu (quantitatives) ont été complétées par des enquêtes directes auprès des animateurs ou responsables de ces organisations ( $c f$. Tableau 2 en annexe). Ces entretiens apportent des éléments sur la place et les représentations de la pluriactivité. Enfin, de manière concomitante, ont été menés dans le cadre du PSDR d'autres travaux dans lesquels les pluriactifs cette fois ci (une cinquantaine), étaient interrogés sur le sens qu'ils donnent à leur pluriactivité, et sur leurs motivations à mener leur activité professionnelle de cette manière (BERNARD, 2009 ; D'ALDEGUIER, 2010 ; FIORELli et al., 2011 ; TALLON, 2011a). Cet ensemble de données permet de confronter des points de vue, pour en repérer les convergences ou les divergences susceptibles d'avoir un impact dans la dynamique territoriale de soutien à la pluriactivité.

Revenons au contexte régional pour saisir les enjeux du soutien à la pluriactivité. Le Languedoc-Roussillon est une région contrastée en termes d'activités, faisant preuve d'un dynamisme économique et d'un pouvoir d'attraction toujours très important, tout en montrant de forts indices de précarité : la région se classe au tout premier rang pour la création nette d'emplois, mais elle subit simultanément un taux de chômage élevé : 12,2\% au premier trimestre 2009 (RocHE, 2009). L'activité de ses territoires ruraux est fragilisée par les crises sectorielles agricoles, crise viticole en particulier. La pluriactivité constitue une activité largement et de tous temps pratiquée, mais son poids et son impact actuels sont toujours mal évalués. De nombreuses structures d'accompagnement s'intéressent à la question, et la pluriactivité commence à être déclinée dans les politiques institutionnelles. Le Conseil régional a d'ailleurs fait de la pluriactivité un de ses axes d'intervention et a mené de 2006 à 2008 une expérimentation portant sur l'accompagnement à la pluriactivité (POMBO, 2008). Le Haut-Languedoc, sur lequel porte notre focus, est constitué des communes du Nord-Ouest du département de l'Hérault, communes exclusivement rurales. Ce territoire de moyenne montagne méditerranéenne, fortement déstabilisé par un exode rural sévère, cumule les handicaps structurels (agriculture de montagne, éloignement des pôles urbains, infrastructures limitées...), et n'a que faiblement profité de l'attrait touristique des montagnes méridionales qu'on observe depuis les années 1980. Aujourd'hui l'agriculture, qui n'est plus dominante, est diversifiée, et la pluriactivité occupe une place importante dans l'ensemble des secteurs d'activité. 60\% des exploitations agricoles et $40 \%$ des établissements touristiques (non agricoles) 
sont pluriactifs ou diversifiés (TALLON, 2005). Comme dans le reste de la région, l'accompagnement à la pluriactivité n'existe pas de façon spécifique ni explicite, et les pluriactifs s'insèrent dans les dispositifs courant de la création d'activité. Le dispositif de soutien à la création d'activité du territoire est composé de structures hétérogènes, associations, chambres consulaires et collectivités territoriales de différents rangs, qui interagissent peu, même si elles sont liées par des politiques territoriales communes.

\subsection{Des orientations affichées...}

À l'échelle de la région Languedoc-Roussillon, se dégagent de l'analyse des documents de communication et des entretiens quatre grands registres de justification des actions menées en faveur de la pluriactivité : la nécessité économique; le développement d'un nouveau modèle d'activité ; l'épanouissement personnel ; et, en dernier lieu, le droit à l'identité professionnelle (BERNARDINI et PÉREZ CHUECA , 2009).

Le registre de justification le plus courant est celui de la nécessité économique. Le pluriactif est considéré par l'accompagnateur comme un individu « dans le besoin ", défini par sa catégorisation sociale: allocataire du revenu de solidarité active (RSA), chômeur, femme, agriculteur en difficulté, etc. Nous retrouvons dans cette liste les catégorisations de la précarité. La pluriactivité est pensée en référence à la perte des protections entrainée par les mutations du contexte salarial actuel. Le deuxième registre est, à l'inverse, celui de l'émergence d'un modèle d'activité économique, et le pluriactif est perçu comme un entrepreneur innovateur ou un agent économique du territoire. Qu'elle soit héritée ou au contraire marqueur d'une nouvelle économie, la pluriactivité est pensée en référence au territoire. Le troisième registre est celui de l'épanouissement personnel par l'activité. Le pluriactif est « une mine de talent ", polyvalent et multi-compétent, qui agrège vie professionnelle et vie familiale. Les « néo-ruraux », non issus du milieu agricole et souhaitant s'installer en agriculture, en sont une des figures principales : ayant des parcours professionnels souvent conséquents, ils sont à même de mobiliser des compétences multiples et très éloignées, tout en affirmant dans leur " projet de vie » leurs motivations existentielles. Enfin le dernier registre concerne la sphère juridique et règlementaire. La pluriactivité est un droit auquel on peut prétendre, et l'accompagnement se focalisera particulièrement sur la levée des entraves à l'exercice de la pluriactivité Ces quatre registres interagissent et rares sont les accompagnateurs ne déclinant que l'un d'eux, mais la plupart du temps un registre domine dans les propos énoncés.

À l'échelle du Haut-Languedoc, le lien au territoire apparaît clairement dans la justification de la pluriactivité. Avant de regarder comment les organisations se positionnent spécifiquement par rapport à la pluriactivité, nous avons observé les orientations dominantes de développement mises en avant par chacune d'entre elles.

Les documents écrits de communication laissent apparaitre trois orientations d'intervention principales, une orientation d'ordre économique, une orientation d'ordre social et une orientation d'ordre territorial. Plus ponctuellement on peut trouver des orientations d'ordre environnemental, éducatif et réglementaire. Les 
organisations ont été classées selon leur registre dominant, sachant qu'elles ne se situent jamais dans un registre unique. Le tableau 1 ci-dessous montre qu'elles se distinguent très nettement selon leur place dans le dispositif d'accompagnement : d'un côté structures de développement en charge d'un projet global de territoire, de l'autre côté structures d'accompagnement en relation directe avec les créateurs d'activité.

\section{Tableau 1 - Poids dominant des orientations d'intervention relevées dans les documents de communication des organisations du dispositif d'accompagnement du Haut-Languedoc (DUBIEN, 2010)}

\begin{tabular}{|c|c|c|}
\hline Orientation dominante & $\begin{array}{c}\text { Structures de } \\
\text { développement } \\
\text { territorial }\end{array}$ & $\begin{array}{c}\text { Structures } \\
\text { d'accompagnement }\end{array}$ \\
\hline Économique & $\begin{array}{c}\text { Conseil Régional } \\
\text { Pays Haut Languedoc et } \\
\text { Vignobles } \\
\text { Communauté de } \\
\text { Communes du Minervois } \\
\text { Communauté de } \\
\text { Communes de la } \\
\text { Montagne du Haut } \\
\text {-Languedoc } \\
\text { Communauté de } \\
\text { communes Orb et Jaur } \\
\text { Communauté de } \\
\text { Communes Coteaux et } \\
\text { Châteaux }\end{array}$ & $\begin{array}{c}\text { Boutique de Gestion } \\
\text { AIRDIE } 34 \\
\text { ADEAR LR } \\
\text { Chambre de Commerce et } \\
\text { d'Industrie } \\
\text { Chambre de Métier et } \\
\text { d'Artisanat } \\
\text { Groupement d'Employeurs } \\
\text { de l'Orb }\end{array}$ \\
\hline Sociale & $\begin{array}{c}\text { Conseil général de } \\
\text { I'Hérault } \\
\text { RTAIE Capestang } \\
\text {-Bédarieux (CG34) } \\
\text { Régie de Développement } \\
\text { Local }\end{array}$ & $\begin{array}{c}\text { AIRDIE } 34 \\
\text { ADEAR LR } \\
\text { ARIAC } \\
\text { CEMEA } \\
\text { Terres Vivantes } \\
\text { Énergies alternatives } \\
\text { IFAD Lodève } \\
\text { Pôle emploi } \\
\text { Mutualité Sociale Agricole } \\
\text { Languedoc } \\
\text { Point Info Installation } 34 \\
\text { Solidarité Paysans LR }\end{array}$ \\
\hline Territoriale & $\begin{array}{c}\text { Parc Naturel Régional du } \\
\text { Haut-Languedoc }\end{array}$ & $\begin{array}{c}\text { Accueil Paysan } \\
\text { Racines } 34 \\
\text { Chambre d'Agriculture de } \\
\text { I'Hérault }\end{array}$ \\
\hline Environnementale & & CIVAM Bio Hérault \\
\hline Réglementaire & DDTM & ADASEA 34 \\
\hline
\end{tabular}

Mis à part le Conseil Général, qui colle à ses compétences obligatoires en se focalisant sur la gestion du social, les structures de développement territorial (Communautés de communes, Pays, Parc naturel régional) mettent en avant des orientations d'ordre économique explicitement basées sur l'appui aux entreprises. Ces orientations sont exprimées dans les documents d'objectifs par des propositions 
telles que "s'impliquer dans la création d'entreprise en étant un acteur économique de garantie et de soutien ", ou encore "initier au sein du Pays une mission pour la prospection d'accueil d'entreprises ». Le territoire en tant que tel n'est dominant que pour le Parc naturel régional du Haut-Languedoc : "agir pour l'équilibre de l'occupation de l'espace et de la biodiversité ». Étonnamment ce sont les structures d'accompagnement de projets individuels qui reprennent la thématique du développement équilibré des territoires : « intégrer au mieux l'agriculture dans la gestion des territoires ruraux et périurbains ", ou encore " être facteur de développement local en maintenant la vie en milieu rural ». C'est cependant dans le registre social que se situe la majorité des structures d'accompagnement du territoire. Elles ont en effet comme mission d'accompagner un individu dans la mise en place de son activité. Elles communiquent autour des apports du projet pour la personne et sur leur propre rôle dans l'appui au parcours de création, en particulier lorsque la situation est délicate : " accompagner ses ressortissants dans les crises qu'ils subissent ». Par des messages courts elles mettent en avant leurs valeurs, qui sont par ailleurs stratégiquement des marques de différenciation. Une telle se définit par l'autonomisation de l'individu : " soutenir toute initiative qui s'inscrit dans une démarche autonome et solidaire ", d'autres par leurs dynamiques collectives : " impulser un nouveau cadre de travail qui favoriserait l'initiative dans un cadre collectif ", "faire s'entraider des publics différents ", qui peuvent par ailleurs être territoriales : "contribuer à la cohésion sociale et territoriale ».

\section{2. ... différentes des pratiques...}

Compte tenu de ces éléments, on pourrait s'attendre à trouver des modes de prise en charge et une reconnaissance de la pluriactivité allant dans le sens des orientations de la structure. Or l'analyse des enquêtes montre que les liens sont difficiles à établir.

Tout d'abord, les structures enquêtées répondent avec hésitation quand on leur demande la définition qu'elles donnent de la pluriactivité. Certaines ne savent pas : « on n'a pas de définition précise... »; «j'en ai pas une ... ah c'est difficile...» ; « une société qui ... qui diversifie ses activités .... et qui se lance dans autre chose pour compléter ses revenus la plupart du temps je suppose, pour faire que l'activité première continue de ... d'exister en fait ». D'autres enquêtés contournent le mot lui-même : « en fait on l'utilise pas forcément le terme pluriactivité mais, ... ça recouvre en fait une réalité »; « on n'appelle pas vraiment ça pluriactivité, on appelle ça diversification "; " ça dépend si on parle de multiactivité ou de pluriactivité ". Quatre structures disent ne pas avoir de définition de ce terme : une communauté de communes, la Direction Départementale des Territoires et de la Mer, le PNR HL et Pôle emploi.

Pour les autres structures enquêtées, au-delà des hésitations relevées, trois références sont utilisées pour définir la pluriactivité : l'entreprise, l'activité et l'agriculture. Pour les structures faisant référence au monde de l'entreprise et à ses statuts, il s'agit plutôt d'avoir plusieurs fonctions au sein de la même entreprise grâce à des compétences multiples, ou bien d'associer une activité indépendante à une activité exercée sous un autre statut. 
Pour les structures se référant à l'activité, la pluriactivité correspond à une combinaison de plusieurs "métiers ", identifiables par les compétences et la sphère professionnelle auxquelles il réfère. Cette définition a été citée par des coopératives d'activités et d'emploi, des structures d'insertion, et une structure consulaire. Enfin la dernière définition de la pluriactivité, la plus couramment citée par les structures enquêtées, se réfère à l'agriculture. Ces structures sont essentiellement à vocation agricole (Organismes Professionnels Agricoles, associations), ou sont des structures en charge d'un projet de territoire décliné à une échelle locale (Communautés de communes fortement marquées par l'agriculture). Cette définition se compose de deux sous-définitions et permet de distinguer les structures pour lesquelles la pluriactivité est assimilée à une activité de diversification agricole (activités d'hébergement, de transformations des produits, de vente directe) ou en est tout à fait distincte. Concrètement, pour la première catégorie, un éleveur ayant un camping à la ferme n'est pas pluriactif, alors que dans le deuxième cas il l'est.

Difficile à définir, la pluriactivité semble tout aussi difficile à évaluer. Peu de structures enquêtées arrivent à se prononcer sur les évolutions de la pluriactivité, par manque de données exploitables : "dire que ça a augmenté, ça a baissé, je ne vais pas m'aventurer ", " est-ce qu'il y en a plus en pluriactivité qu'il y en avait avant ? ... ça ne m'a pas frappé !! ». Par contre, sur ce territoire divers facteurs sont perçus comme autant d'incitations à la pluriactivité : la crise de l'emploi en général ou la crise viticole en particulier (53\% des structures y font mention explicitement), la présence de petites structures, agricoles ou non (17\% des réponses), la précarité économique (23\% des réponses).

La diversification apparaît comme une alternative évidente voire comme une évolution des modes de conduite de l'activité : " [de plus en plus] les agriculteurs, les petites structures voient la nécessité de se diversifier »; " avec la crise, les gens ont plus tendance à diversifier leur offre, sur leur même secteur d'activité, sur une même branche "; " maintenant, on voit beaucoup d'activités secondaires, de plus en plus, les artisans proposent un panel de services et la multiactivité, ça rejoint aussi le multiservices ". Cependant la pluriactivité est, en elle-même, très largement considérée comme un mal nécessaire et un symptôme de crise : "avec la crise viticole, les viticulteurs se tournent vers la pluriactivité par contrainte ... l'obligation est alimentaire : on est dans une époque de crise, au sens large "; "la nécessité économique est un moteur à la pluriactivité »; "il y a peu d'activités donc les personnes s'orientent à défaut vers une création d'activité "; " ce n'est pas une activité forcément viable ... combiner deux activités et arriver à durer, il n'y en a pas souvent [qui réussissent]"; " moi, j'ai une vision assez pessimiste du territoire on se confronte [en matière de pluriactivité] à ... légalement comment on peut faire ?" ; "peut-être mettre en œuvre le maximum de ses compétences pour s'en sortir ?».

Peu de structures considèrent la pluriactivité comme une opportunité et sortent du registre pessimiste et fataliste de la pluriactivité marqueur de précarité, mais la pluriactivité parait somme toute naturelle : « est-ce que ça va augmenter ... j'ai pas non plus vraiment constaté de grosse tendance, mais il y en aura toujours parce que c'est normal d'envisager son projet comme ça » ; " oui, je pense que [la pluriactivité] 
c'est rentré dans les esprits. Avant ça se disait pas, mais ça se faisait déjà "; " avec la crise qui existe aujourd'hui, la pluriactivité est une mesure qui permet à des personnes, en développant plusieurs petites activités de créer leur activité ». Les registres non économiques ne sont évoqués que par une structure : "il y a aussi, à travers la pluriactivité, la place de la femme...».

Nous pouvons résumer les visions de la pluriactivité repérées dans le Haut Languedoc en cinq points :

1) La pluriactivité est vue comme une situation de transition, permettant une installation agricole progressive, ou de simplement prendre le temps nécessaire au montage d'un projet complexe ou incertain. Elle permet de faire face à une crise (pensée comme ponctuelle). C'est le point de vue des associations d'accompagnement agricole ou de celles qui accompagnent un public dit précaire (PII, Airdie, CCI, Solidarité Paysan LR, Adear LR, IFAD), ainsi que d'une communauté de communes dans laquelle les personnes allocataires du RSA sont nombreuses, la CC OJ.

2) La pluriactivité est vue comme une complémentarité, à la fois dans la mobilisation de compétences et dans la conduite des activités. Là encore, nous retrouvons des associations, dont les orientations sociales dominent : Racines 34, BG, Ariac, MSA, Adasea Hérault, ADTV 34.

3) La pluriactivité est vue comme la possibilité de maintenir l'agriculture, les activités et les personnes en milieu rural (dans un enjeu de développement des campagnes), en confortant les entreprises dans leur contexte territorial, et en stabilisant socialement les personnes. Nous retrouvons dans cette catégorie la majeure partie des collectivités territoriales, ainsi que les associations très ancrées dans leur territoire : CR, Pays HLV, GE d'Orb, CA, Accueil Paysan, RDL, CEMEA/ARIES, CC Montagne HL, Adear LR, MSA, CG-Département économie, CG-RTAIE, ADTV 34, CC côteaux et châteaux.

4) La pluriactivité est vue comme la possibilité de réaliser un projet de vie, dans le choix de vie en milieu rural et la symbiose de l'activité avec le territoire et les choix familiaux. Elle permet l'intégration sociale des néo-ruraux. Pour le coup, dans cette catégorie, on ne trouve aucune structure dont l'orientation dominante est économique. Mis à part la structure d'insertion du Conseil général (le RTAIE), les autres structures sont des associations fortement positionnées dans les démarches d'économie sociale et solidaire: Accueil Paysan, CG-RTAIE, ADTV 34, Énergies Alternatives.

5) Enfin la pluriactivité est vue comme un frein important au développement d'activités. Elle bloque l'accès aux mesures de droit commun (Dotation Jeune Agriculteur, accès au foncier agricole, permis de construire, etc.), et est incompatible avec une activité de qualité (dispersion). Très minoritaires, les structures de ce groupe se situent dans des orientations économiques ou réglementaires, prenant en compte l'activité avant l'individu : Chambre des métiers, DDTM, Adasea 34, CC OJ. 
Une structure se détache du lot par l'impossibilité de lui reconnaitre une vision dominante : l'association Terres Vivantes 34 (ADTV34). Radicalement positionnée dans l'installation progressive agricole non aidée, elle s'adresse à un public n'entrant pas dans le cadre conventionnel de la création d'activité agricole. Travaillant depuis sa création sur les questions de pluriactivité, elle est fortement identifiée par la quasi-totalité des structures enquêtées comme LA ressource territoriale en matière de pluriactivité.

\section{3. ... mais aussi peu partagées par les pluriactifs eux-mêmes}

Pourtant, lorsqu'on interroge les pluriactifs, et non pas leurs accompagnateurs, le sens que prend pour eux la pluriactivité est nuancé. L'intérêt que leur apporte la mise en œuvre de diverses compétences est central : « la pluriactivité est une combinaison d'activités différentes, pas forcément toutes rémunérées et qui demandent des compétences différentes ». Nous sommes dans le registre de l'épanouissement personnel, parfois au détriment assumé de la sécurité matérielle : « la pluriactivité c'est être pris par plusieurs passions, activités, et ne pas vouloir les choisir » ; "c'est complètement, totalement égoïste, c'est à dire je me fais plaisir, je gère mon temps, [...] j'ai besoin de vivre d'autre chose à côté sinon je peux pas avancer c'est pas possible ", « j'aime tout quoi...et j'ai toujours fait plein de trucs d'ailleurs ma mère disait toujours : 'bonne à tout, bonne à rien' ». L'adaptation à la situation économique du territoire est une des motivations qui conduit à multiplier ses activités : " honnêtement c'est un peu obligé dans un... endroit aussi reculé », mais elle résulte d'un choix engagé : « au départ l'installation s'est faite ici. [...] Ce n'était pas l'activité elle-même qui était visée mais plutôt prendre un territoire vierge et l'amener ou le ramener à la vie ». Les réponses des pluriactifs enquêtés montrent que la pratique de la pluriactivité et la volonté de vivre sur un territoire sont intimement liées : " je suis complètement passionné du coin dans le sens où c'est un espace où j'ai l'impression de vivre une aventure tous les jours ». Si la pluriactivité n'apparaît pas toujours comme un choix, mais comme une contrainte pour faire face à un problème économique sérieux, ce ressenti négatif de la pluriactivité reste toutefois minoritaire. Il n'est formulé que par des personnes dont le système d'activité s'est construit sur des ruptures nécessitant un rééquilibrage rapide, et dont la pluriactivité n'a pas été envisagée en amont mais s'est imposée pour répondre à des exigences spécifiques non attendues (BERNARD, 2009).

\section{-4 - \\ La divergence des points de vue, ou le " conflit des mondes »}

Comment au filtre de ces entretiens, expliquer le flou qui perdure toujours autour d'une pratique courante en Languedoc-Roussillon, de plus en plus médiatisée et largement débattue au moins dans la profession agricole ? Comment interpréter les disjonctions entre les projets mis en avant par les organisations de soutien à la création d'activité, qu'ils soient de natures économique, sociale ou territoriale, et leur 
faible reconnaissance de la pluriactivité ? Ou encore les différences entre le ressenti des apports de la pluriactivité par l'individu et par les structures d'accompagnement ?

Dans le Haut-Languedoc l'influence des dynamiques économiques négatives du territoire est visible. Les organisations d'accompagnement du Haut-Languedoc semblent avoir des visions relativement limitées des apports possibles de la pluriactivité sur l'évolution économique du territoire. On n'y retrouve pas la palette des justifications mises en avant à l'échelle de la région Languedoc-Roussillon par les accompagnateurs. Recentrée autour de sa capacité de résilience qui en fait un atout dans la gestion des crises, la pluriactivité reste le vecteur du " petit », associé à l'idée de « fragile ». Les situations de précarité bien visibles et en développement dans le Haut-Languedoc forment bien souvent un filtre au travers duquel la pluriactivité n'est comprise qu'en négatif d'une crise.

Pourtant, le lien fait par la grande majorité des structures interrogées entre pluriactivité et vie des territoires ruraux semble assez consensuel, et le maintien d'un secteur agricole, corrélé à l'accueil de nouvelles populations dans une zone rurale vue comme " difficile ", mobilise tous les responsables du développement territorial. Que le Conseil régional et le Conseil général se rejoignent sur cette question montre la préoccupation qui émerge quant à l'accompagnement des arrièrepays vers un développement non plus pensé uniquement en termes de périphéries d'espaces urbains ou périurbains, mais pensé en termes de territoires dont les dynamiques endogènes priment. Cependant des divergences dans les visions de la pluriactivité et du rôle qu'elle peut jouer dans le développement d'activités rurales demeurent, et les coordinations entre organisations apparaissent de ce fait parfois compromises. Comme nous l'avons vu, certaines structures enquêtées ne définissent pas la pluriactivité. Celle-ci peut ne pas correspondre à une réalité prise en compte et travaillée dans la structure, lorsque n'est prise en considération dans le projet de la personne que l'activité principale (cas de la DDTM ou de Pôle emploi). Mais lorsque les « documents projet » de la structure y font explicitement référence, comme c'est le cas pour le Parc naturel régional du Haut-Languedoc, cette contradiction, à savoir ne pas être capable de définir ce qu'on promeut, est pour le moins surprenante. En matière agricole, la pluriactivité souffre toujours de l'image poussiéreuse qu'elle traine depuis les années 1960 d'une agriculture peu productive et dispersée, ce qui rend prudentes les organisations professionnelles agricoles ou de développement rural du territoire quant à sa promotion.

Malgré tout le Haut-Languedoc n'est pas coupé de son espace régional, et la démarche innovante menée par le Conseil régional en matière de pluriactivité représente à beaucoup d'égards une nouveauté dans le champ des politiques économiques régionales qui devrait faire évoluer les formes d'accompagnement. Un « dispositif régional d'accompagnement des entrepreneurs pluriactifs en milieu rural ", baptisé "Pluriactiv Rural ", est en phase de test depuis 2011. Faisant suite à trois ans d'expérimentation, il devrait mener à réorienter la politique régionale d'appui à la pluriactivité, dans la mesure où les partenaires se saisiront de cette opportunité. Mais ce programme est d'ores et déjà en partie limité par son cadre d'action unique: il ne laisse pas de place à la reconnaissance des 
spécificités territoriales, alors même que la région Languedoc-Roussillon est une mosaïque de contextes et d'enjeux territorialisés, qui donnent forme à la pluriactivité (GASSELIN, 2011). Ainsi la pluriactivité des viticulteurs en crise dans le Minervois n'a pas grand-chose à voir avec la pluriactivité des agriculteurs des moyennes montagnes méridionales où elle s'impose localement, dans sa reconnaissance sociale et institutionnelle, comme un mode de vie. Il n'est donc pas certain que le souffle impulsé par la dynamique du Conseil régional trouve un écho sur un territoire comme le Haut-Languedoc, dans lequel les relais régionaux sont très discrets. D'autant plus que les espaces d'expression des pluriactifs, qui peuvent exister ponctuellement en région et sur le territoire dans les dispositifs d'accompagnement, sont en partie brouillés par les divergences de postures liées à la pluriactivité. Le HautLanguedoc, dans lequel l'héritage pluriactif est métissé de stratégies contemporaines d'adaptation aux contraintes et aux ressources territoriales (TALLON et al., 2009), est représentatif d'espaces périphériques toujours à la recherche de leurs propres leviers de développement (DERIOZ, 2010). S'il y a bien dans le Haut-Languedoc une stratégie de développement territorial, qui se traduit par un dispositif de soutien à la création d'activité en partie territorialisé (via le dispositif d'insertion du Conseil général), on ne peut pas voir de volonté partagée entre acteurs territoriaux (Pays du HautLanguedoc et Vignobles, Parc naturel régional du Haut-Languedoc, Communautés de communes et leurs partenaires) pour faire de l'accompagnement à la pluriactivité un thème majeur et fédérateur du développement.

\section{-5 - Conclusion}

Nos observations dans le Haut-Languedoc montrent que la pluriactivité est relativement occultée, et que les actions qui s'y réfèrent ne sont pas valorisées. Il n'y a pas de stratégie territoriale de gestion des pluriactivités, et cela malgré l'enjeu majeur, largement énoncé par la grande majorité des structures interrogées, d'adaptation ou de réponse à la double crise de ce territoire. Nous retrouvons, à l'intérieur du dispositif d'accompagnement, les ambigüités de positionnement des secteurs professionnels et des organisations d'accompagnement vis-à-vis de la pluriactivité. Or les divergences qui peuvent provenir des différentes perceptions du projet pluriactif par les structures en charge de l'accompagnement et par le porteur lui-même, peuvent avoir un effet contraignant et freiner le développement des activités sur un territoire. À travers l'idée d'accompagnement se pose la question du projet, projet individuel qui croise le projet territorial et qui prolonge ou s'écarte des modèles sociaux ou économiques en place. L'accompagnement à la pluriactivité met en jeu, dans une relation triangulaire, l'individu, l'accompagnateur et la société via l'institution dont dépendra l'accompagnateur, et exige ainsi une reconnaissance mutuelle a minima des modèles et des valeurs portés par les uns et les autres. La question des finalités de l'accompagnement s'exprime au travers des divergences de justifications observées. Car, bien que relativement silencieux, les modèles d'activités contenus dans les formes de pluriactivité s'expriment dans les attendus et les orientations des projets pluriactifs. Surfant sur les paradoxes (TALLON, 2011a), les modèles de travail 
pluriactifs des zones rurales poussent fortement les accompagnateurs à s'interroger sur les fondements idéologiques de leurs représentations du travail. Car requalifier le travail ne peut en aucune manière être un acte neutre dans le contexte actuel de transformation de nos sociétés salariales. Dans les choix d'accompagnement qui leur sont proposés, les pluriactifs se retrouvent face à des orientations politiques structurantes. Au sein même des structures d'accompagnement, les tensions dans la coexistence de justifications de la pluriactivité, parfois tout à fait contradictoires, nous mettent en présence d'une pluralité de "mondes", telle que modélisée par BOLTANSKI et THÉVENOT (1991).

Peut-on malgré tout concevoir l'idée d'un dispositif d'accompagnement à la pluriactivité répondant aux attendus des pluriactifs, et fonctionnel, c'est-à-dire basé entre autres sur l'échange, la mutualisation et le travail en réseau d'acteurs situés dans des registres d'action aussi hétérogènes? Comment et sur quoi fonder l'accord, pour reprendre les termes de BOLTANSKI et THÉVENOT (1991) ?

Rappelons qu'un univers soumis à la contrainte de justification est un univers soumis à l'épreuve de la critique, et que celle-ci ne peut se déployer que dans la reconnaissance de la coexistence de plusieurs mondes : "La prise en compte de plusieurs mondes permet de préciser la contrainte de justification. Les personnes n'y seraient pas soumises si elles n'étaient affrontées à la critique. La possibilité de sortir de la situation présente et de la dénoncer en prenant appui sur un principe extérieur et, par conséquent, la pluralité des mondes, constituent donc la condition d'une action justifiée. " (BOLTANSKI et THEVENOT, 1991). Un litige prendra forme lorsqu'il y a désaccord et contestation de l'ordonnancement de la situation. Le litige peut être réglé par l'épreuve, dans laquelle sera convoqué un " principe supérieur commun ", qui rétablira les grandeurs relatives des acteurs impliqués. Mais lorsque ce principe supérieur commun est mal identifié, le processus ne peut arriver à terme. Les "situations troubles » ou l'ambigüité des " assemblages composites" comportant des êtres relevant de plusieurs mondes, suscitent chez les acteurs un sentiment d'inquiétude et de malaise vis-à-vis de l'épreuve. Dans ces cas précis, les différends sont réglés par le recours au compromis, par lequel les parties s'accordent pour composer, c'est à dire suspendent le différend sans passer par l'épreuve. De fait, la contrainte de justification est elle aussi suspendue. Cependant, le compromis ne peut exister sans présupposé d'un bien commun vers lequel les protagonistes de l'accord tendent. Ce bien commun est par ailleurs difficile à clarifier, et son indétermination rend fragile le compromis, qui peut n'apparaître que comme un agencement non fondé. Dans notre cas, nous voyons que le simple fait de s'accorder sur une définition de la pluriactivité est impossible, tant ce sont les frontières même des secteurs d'activité qui sont menacées. Et peut-être est-ce le propre d'une pratique d'activité complexe, en référence au modèle de travail français, de se déployer dans une palette de mondes dont la référence commune, la définition du travail, nous échappe aujourd'hui ?

Les dispositifs d'accompagnement à la pluriactivité en émergence en LanguedocRoussillon et en France, et de manière plus large les politiques publiques appliquées à la pluriactivité se cherchent une légitimité, une voie pour se construire. Les compromis 
ponctuellement mis en place au gré des territoires, des orientations politiques locales ou nationales, des évolutions réglementaires, des rapports de force entre acteurs, ouvrent des possibilités d'action, malgré les difficultés de fond. Cette suspension « le temps de voir » des différends, résolus ponctuellement par des accords sectoriels ou des arrangements territoriaux aussi variés que non généralisables, témoigne de la volonté d'expérimenter des configurations nouvelles dans l'activité des territoires ruraux. C'est en cela que le repérage des divergences entre acteurs, loin d'être un frein aux politiques territoriales, est dans ce cas un gage de construction de compromis qui permettront l'engagement dans l'action.

\section{$-6-$}

\section{Annexe}

\section{Tableau 2 - Présentation sommaire des organisations enquêtées}

\begin{tabular}{|c|c|}
\hline STRUCTURE & PRÉSENTATION SOMMAIRE \\
\hline Accueil Paysan & $\begin{array}{l}\text { Association de « paysans » qui accompagne la mise en place et } \\
\text { la promotion d'un accueil touristique et social dit " paysan ». } \\
\text { Échelle d'action régionale }\end{array}$ \\
\hline ADASEA & $\begin{array}{l}\text { Association qui a un rôle d'interface entre les agriculteurs, } \\
\text { l'administration et les collectivités territoriales: elle informe, } \\
\text { conseille et accompagne les agriculteurs dans l'élaboration de } \\
\text { leurs dossiers d'aides publiques. Échelle d'action } \\
\text { départementale }\end{array}$ \\
\hline ADEAR LR & $\begin{array}{l}\text { Associations qui rassemblent des « paysans » et d'autres } \\
\text { acteurs du milieu rural. Elles accompagnent des projets } \\
\text { individuels et collectifs qui permettent le développement de } \\
\text { l'agriculture paysanne. Liées à la Confédération Paysanne. } \\
\text { Échelle d'action régionale }\end{array}$ \\
\hline AIRDIE & $\begin{array}{l}\text { Association de financement solidaire. Accompagne les } \\
\text { créateurs d'activité dans leur dossier de financement. Échelle } \\
\text { d'action régionale }\end{array}$ \\
\hline ARIAC & $\begin{array}{l}\text { Coopérative d'activité et d'emploi qui accompagne la création } \\
\text { d'activité en permettant le test préalable des activités dans le } \\
\text { cadre d'un statut de " salarié-entrepreneur ". Échelle d'action } \\
\text { Hauts Cantons (nord-ouest du département de l'Hérault) }\end{array}$ \\
\hline $\begin{array}{l}\text { Boutique de Gestion du } \\
\text { Biterrois }\end{array}$ & $\begin{array}{l}\text { Association qui a pour objectif d'accompagner la création } \\
\text { d'activité et de soutenir le développement des petites } \\
\text { entreprises. Échelle d'action Hauts Cantons et agglomération } \\
\text { de Béziers (34) }\end{array}$ \\
\hline $\begin{array}{l}\text { Communauté de } \\
\text { Communes Coteaux et } \\
\text { Châteaux }\end{array}$ & $\begin{array}{l}\text { Collectivité territoriale dont les compétences obligatoires } \\
\text { sont le développement économique et l'aménagement de } \\
\text { l'espace. Certaines communes adhèrent à des syndicats de cru } \\
\text { selon l'appellation présente sur leur territoire (viticulture). } \\
\text { Échelle d'action : communes du pourtour de St Chinian (34) }\end{array}$ \\
\hline
\end{tabular}




\begin{tabular}{|c|c|}
\hline $\begin{array}{l}\text { Communauté de } \\
\text { Communes Le Minervois }\end{array}$ & $\begin{array}{l}\text { Collectivité territoriale dont les compétences obligatoires sont } \\
\text { le développement économique et l'aménagement de l'espace. } \\
\text { Échelle d'action : communes du pourtour d'Olonzac (34) }\end{array}$ \\
\hline $\begin{array}{l}\text { Communauté de } \\
\text { Communes Montagne } \\
\text { du Haut Languedoc }\end{array}$ & $\begin{array}{l}\text { Collectivité territoriale dont les compétences obligatoires } \\
\text { sont l'aménagement du territoire et les technologies de } \\
\text { l'information et de la communication(TIC). Échelle d'action : } \\
\text { communes du pourtour de La Salvetat (34) }\end{array}$ \\
\hline $\begin{array}{l}\text { Communauté de } \\
\text { Communes Orb et Jaur }\end{array}$ & $\begin{array}{l}\text { Collectivité territoriale dont les compétences obligatoires sont } \\
\text { le développement économique et l'aménagement de l'espace. } \\
\text { Échelle d'action : communes du pourtour d'Olargues (34) }\end{array}$ \\
\hline $\begin{array}{l}\text { Chambre de Commerce } \\
\text { et d'Industrie }\end{array}$ & $\begin{array}{l}\text { Établissement consulaire dont l'objectif est de contribuer au } \\
\text { développement économique du territoire par le conseil direct } \\
\text { aux entreprises et aux créateurs d'activité des secteurs du } \\
\text { commerce et de l'industrie, l'animation et la fédération des } \\
\text { acteurs économiques, la représentation des professionnels. } \\
\text { Échelle d'action départementale }\end{array}$ \\
\hline CEMEA/ARIES & $\begin{array}{l}\text { Association qui a pour objectif d'accompagner les artistes en } \\
\text { insertion à la mise en place de leur projet, en particulier via } \\
\text { des projets collectifs. Échelle d'action : Ouest du département } \\
\text { de l'Hérault }\end{array}$ \\
\hline $\begin{array}{l}\text { Chambre d'Agriculture } \\
\text { de l'Hérault }\end{array}$ & $\begin{array}{l}\text { Établissement consulaire qui a pour objectif l'animation et le } \\
\text { développement des territoires ruraux. Elle conseille les } \\
\text { créateurs d'activité agricole et les exploitations agricoles en } \\
\text { place. Échelle d'action départementale }\end{array}$ \\
\hline $\begin{array}{l}\text { Chambre de Métier et } \\
\text { d'Artisanat de l'Hérault }\end{array}$ & $\begin{array}{l}\text { Établissement consulaire qui a pour vocation de représenter } \\
\text { les intérêts généraux de l'artisanat auprès des pouvoirs publics. } \\
\text { Conseil direct aux artisans et créateurs d'activité. Échelle } \\
\text { d'action départementale }\end{array}$ \\
\hline FD CIVAM 34 & $\begin{array}{l}\text { Association qui accompagne les porteurs de projet en milieu } \\
\text { rural, de l'idée au projet. Elle est un relais vers les structures } \\
\text { œuvrant dans les différents domaines concernés (tourisme, } \\
\text { agriculture...). Échelle d'action départementale }\end{array}$ \\
\hline $\begin{array}{l}\text { Conseil général de } \\
\text { l'Hérault }\end{array}$ & $\begin{array}{l}\text { Collectivité territoriale en charge des domaines de l'éducation } \\
\text { (collèges), des transports, du logement, de la culture, de } \\
\text { l'action sociale. Élabore les politiques publiques et } \\
\text { accompagne leur mise en œuvre. Échelle d'action } \\
\text { départementale }\end{array}$ \\
\hline $\begin{array}{l}\text { Conseil régional } \\
\text { Languedoc-Roussillon }\end{array}$ & $\begin{array}{l}\text { Collectivité territoriale en charge l'élaboration et de la mise en } \\
\text { œuvre de la politique d'aménagement du territoire et de } \\
\text { développement économique, de la formation professionnelle, } \\
\text { de l'éducation, des transports. Élabore les politiques publiques } \\
\text { et accompagne leur mise en œuvre. Échelle d'action régionale }\end{array}$ \\
\hline $\begin{array}{l}\text { Direction } \\
\text { Départementale des } \\
\text { Territoires et de la Mer }\end{array}$ & $\begin{array}{l}\text { Établissement public sous l'autorité du Préfet de l'Hérault. II } \\
\text { anime et met en œuvre les politiques du ministère de } \\
\text { l'Écologie, de l'Énergie, du Développement Durable et de la } \\
\text { Mer (MEEDDM) et du ministère de l'Agriculture, de } \\
\text { l'Alimentation et de la Pêche (MAAP). Échelle d'action } \\
\text { départementale }\end{array}$ \\
\hline
\end{tabular}




\begin{tabular}{|c|c|}
\hline Énergies alternatives & $\begin{array}{l}\text { Coopérative d'Activités et d'Emploi qui a pour objectif } \\
\text { d'accompagner la création d'activité. La CAE héberge des } \\
\text { entrepreneurs-salariés en « test ", et des } \\
\text { entrepreneurs-associés. Échelle d'action : zone littorale de } \\
\text { l'Hérault et Sud des Hauts Cantons }\end{array}$ \\
\hline $\begin{array}{l}\text { Mutualité Sociale } \\
\text { Agricole Languedoc }\end{array}$ & $\begin{array}{l}\text { Mutualité qui gère la protection sociale des salariés et des } \\
\text { non-salariés de l'agriculture. A ce titre elle accompagne les } \\
\text { agriculteurs en difficulté. Échelle d'action départementale }\end{array}$ \\
\hline $\begin{array}{l}\text { Pays Haut-Languedoc } \\
\text { et Vignobles }\end{array}$ & $\begin{array}{l}\text { Syndicat mixte. Structure intercommunale en charge d'un } \\
\text { projet de territoire. Le Pays articule ses actions autour des } \\
\text { thèmes suivant : habitat, environnement, tourisme, } \\
\text { patrimoine, services, culture et communication, et économie. II } \\
\text { accompagne directement les créateurs d'activité. Échelle } \\
\text { d'action : Hauts Cantons }\end{array}$ \\
\hline $\begin{array}{l}\text { Parc Naturel Régional } \\
\text { du Haut-Languedoc }\end{array}$ & $\begin{array}{l}\text { Syndicat mixte. Structure intercommunale en charge d'un } \\
\text { projet global de territoire portant sur l'environnement et la } \\
\text { biodiversité, I'agriculture, la diversification, le patrimoine. } \\
\text { Échelle d'action : partie nord des Hauts Cantons }\end{array}$ \\
\hline Point Info Installation & $\begin{array}{l}\text { Association émanant des Jeunes Agriculteurs. Service labellisé } \\
\text { par la DDTM sur avis de la CDOA dont l'objectif est } \\
\text { d'accompagner les porteurs de projet en agriculture jusqu'à } \\
\text { leur installation. Échelle d'action départementale }\end{array}$ \\
\hline $\begin{array}{l}\text { Pôle emploi } \\
\text { Béziers-Boujan }\end{array}$ & $\begin{array}{l}\text { Établissement public chargé de l'inscription et de l'orientation } \\
\text { des demandeurs d'emploi et du versement des allocations. } \\
\text { Échelle d'action Nord-Ouest du département }\end{array}$ \\
\hline Racines 34 & $\begin{array}{l}\text { Association qui fédère un réseau d'accueil éducatif à la ferme, } \\
\text { agréé par l'Éducation Nationale. Accompagne au } \\
\text { développement de l'accueil et des animations « nature » sur } \\
\text { les fermes. Racines } 34 \text { est membre de l'association Racines et } \\
\text { des CIVAM. Échelle d'action départementale }\end{array}$ \\
\hline $\begin{array}{l}\text { Régie de } \\
\text { Développement Local }\end{array}$ & $\begin{array}{l}\text { Association relai du Conseil général en charge de la mise en } \\
\text { œuvre des politiques d'insertion du territoire. Elle s'adresse } \\
\text { aux bénéficiaires du RSA, aux jeunes en recherche d'emploi, } \\
\text { aux travailleurs handicapés, et chômeurs de longue durée. } \\
\text { Structure financeur, contrôleur et coordinateur. Échelle } \\
\text { d'action: Hauts Cantons }\end{array}$ \\
\hline $\begin{array}{l}\text { RTAIE Capestang } \\
\text { Bédarieux } \\
\text { (CLI de Capestang et CLI } \\
\text { de Bédarieux / Pézenas) }\end{array}$ & $\begin{array}{l}\text { Collectivité territoriale, service du Conseil général en charge } \\
\text { de la CLI (Commission Locale d'Insertion) de } \\
\text { Capestang-Bédarieux qui réalise l'accompagnement dans } \\
\text { l'emploi de personnes bénéficiaires des minima sociaux. } \\
\text { Échelle d'action : Nord-Ouest du département de l'Hérault }\end{array}$ \\
\hline Solidarité Paysans LR & $\begin{array}{l}\text { Association dont les missions sont l'information et le conseil } \\
\text { aux agriculteurs en difficulté, la lutte contre l'exclusion et la } \\
\text { défense collective des agriculteurs en difficulté. Échelle } \\
\text { d'action régionale }\end{array}$ \\
\hline Terres Vivantes 34 & $\begin{array}{l}\text { Association qui a pour objet l'accompagnement à l'installation } \\
\text { progressive agricole, l'animation de territoires ruraux et le } \\
\text { soutien aux projets collectifs. A sa création elle était liée à la } \\
\text { Confédération Paysanne. Échelle d'action départementale }\end{array}$ \\
\hline
\end{tabular}




\section{Bibliographie}

ASSEDIC des Alpes (2008) La saisonnalité en Tarentaise. Assedic, Albertville.

AubreE P, MAReChal G (2008) Comment accompagner les projets multifonctionnels ? L'expérience du programme Terriam. Cahiers Agricultures 17 (6) : 561-565.

BEFFY M (2006) En 2005, plus d'un million de salariés ont plusieurs employeurs. INSEE Première (1081).

BEL M (2009) Compétences et dynamiques territoriales : quelles interactions? Géographie, économie, société 11 (3) : 213-232.

Belorgey J-M, Fouquet A (dirs.) (2000) Minimas sociaux, revenus d'activité, précarité. La Documentation Française, Paris.

BERNARD C (2009) Quelle est l'incidence des caractéristiques et des ressources du territoire dans la stratégie des pluriactifs ? Mémoire de Master 2 « Gestion des Espaces Ruraux Aménagement et Développement Local ». Université Montpellier 3, Montpellier.

Bernardini S, PÉRez ChueCA A (2009) Analyse de l'émergence de dispositifs d'accompagnement aux systèmes d'activité pluriactifs sur la région du Languedoc Roussillon. Mémoire présenté en vue de l'obtention du diplôme d'ingénieur agronome (Sandra BERNARDINI) et du diplôme Master 2 "Innovation et développement des territoires ruraux" (Alberto PÉREZ CHUECA). AgroCampus Ouest, Rennes, Université de Montpellier 3, Montpellier.

Biche B, Gerbaux F, Le Monnier J, Perret J (1996) Créer son emploi en milieu rural, les chemins de traverse de la pluriactivité. L'Harmattan, Paris.

BLANC M (2003) Les marchés ruraux du travail, vers une spécialisation fonctionnelle des espaces ?. INRA Sciences sociales 2 (1) : 1-4.

BoltANSKI L, THEVENOt L (1991) De la justification, les économies de la grandeur. Gallimard, Paris.

BOUDY J-F (2009) Vivre de deux métiers, la pluriactivité. L'Harmattan, Paris.

Bureau M-C, Perrenoud M, Shapiro R (2009) L'artiste pluriel. Démultiplier l'activité pour vivre de son art. Presses Universitaires du Septentrion, Villeneuve d'Ascq.

Butault J-P, Delame N, Lerouvillois P, Krebs S (1999) La pluriactivité : un correctif aux inégalités du revenu agricole. Économie et statistique, 329-330 : 165-180.

CASAUX L (1993) La pluriactivité ou l'exercice par une même personne physique de plusieurs activités professionnelles. Librairie Générale de droit et de jurisprudence, Paris.

CORCUFF P (1998) Justification, stratégie et compassion : apport de la sociologie des régimes d'action. Correspondances (Bulletin d'information scientifique de I'Institut de Recherche sur le Maghreb Contemporain), (51) : 9.

COUTURIER I (1994) La diversification en agriculture (aspects juridiques). L'Harmattan, Paris.

D'ALDEGUIER G (2010) La question du genre dans la pluriactivité. Comprendre les positions des femmes pluriactives dans le milieu rural en Languedoc Roussillon pour améliorer les dispositifs d'accompagnement locaux. Mémoire présenté en vue de l'obtention du diplôme d'ingénieur agronome. École d'agriculture de Purpan, Toulouse.

DÉRIOZ P (2010) Management of environment and landscapes in moutain areas: social representations, actors and institutions in a regional park. In : VACCARO C, BELTRAN O (eds), Social and ecological history of the Pyrenees : state, market and landscape. Left coast Press Inc, Walnut Creek, Californie, EUA.

DUBIEN A (2010) Identification de registres de justification de la pluriactivité pour l'accompagnement. Application au territoire du Haut-Languedoc héraultais. Mémoire présenté en vue de l'obtention du diplôme d'ingénieur agronome. AgroSup, Dijon.

Fiorelli C, Dufour A, MASSEIN G, SAUVAigo C (2011) Pluriactivité : entre sécurité matérielle et plaisir au travail. Pour 212 : 179-186.

FISCHER BOEL M (2006). Interview. Financial Times, 29 décembre 2006.

FONT E (2000) Les Activités non agricoles dans la recomposition de l'espace rural. L'Harmattan, Paris.

Fouquet A (1998) Travail, emploi et activité. La lettre du Centre d'Études de l'Emploi, 52 : 11. 
GASSELIN P (2011) Co-conception d'une politique publique pour une région arrière du développement : le cas de la pluriactivité en Languedoc-Roussillon. Pour, 212 : 155-163.

Gumuchian H, PeCQueur B (2007) La ressource territoriale. Ed. Anthropos, Paris.

HEINICH N (2008) Régime vocationnel et pluriactivité chez les écrivains : une perspective compréhensive et ses incompréhensions. Socio-logos. Revue de l'association française de sociologie (3) : 7[En ligne URL : http://socio-logos.revues.org/1793], mis en ligne le 28/05/2008, consulté le 21/04/2010.

HeRvieu B, Viard J (1996) Au bonheur des campagnes (et des provinces). Éditions de l'Aube, La Tour d'Aigues.

HOCHART R (2007) «C'est complètement insensé »pour la Confédération paysanne. Agrapresse Hebdo, en ligne [http://www.agrapresse.fr/c-est-compl-tement-insens-pour-la-conf-d-ration-paysanneart223212-24.html], mis en ligne le 8/01/2007, consulté le 20/04/2012.

LAurent C, MOuriaux M-F, Mundler P (2006) Combinaison d'activités professionnelles et multifonctionnalité de I'agriculture : une résurgence de la dimension territoriale. Document de travail du Centre d'Études de l'Emploi, 70.

LAURENT C, MUNDLER P (2006) L'accompagnement de la pluriactivité en question. Les résultats du programme PSDR 2 Territoires, acteurs et agriculture en Rhône-Alpes, en ligne [http://www.psdrra.fr/documents/Ressources/Ressources_PSDR2/Syntheses/Axe3_PSDR_310_std.pdf], consulté le $18 / 11 / 2009$.

LEMÉTAYER J M (2007) Discours de clôture du congrès de la FNSEA du 29 mars 2007 à Marseille. FNSEA, en ligne [http://www.fnsea.fr/sites/webfnsea/pres/congr/2007/070329disclot.aspx], consulté le 20/08/2010.

MORA O (2008) Les nouvelles ruralités à I'horizon 2030. Éditions Quae, Versailles.

MAYAUD J-L (1988) De l'étable à l'établi : permanences des adaptations dans la montagne jurassienne. In : GARRIER G, HuBSCHER R (éds) Entre faucilles et marteaux : pluriactivité et stratégies paysannes. Presses Universitaires de Lyon, Lyon.

MENDRAS H (1976) Les sociétés paysannes. Éléments pour une théorie de la paysannerie. Folio Histoire Gallimard, Paris.

PAGES A (2004) La pauvreté en milieu rural. Presses Universitaires du Mirail, Toulouse.

PAGESSE P. (2006) Lettre ouverte à Mariann Fischer Boel, Commissaire européenne à l'agriculture et au développement rural. In : MOMAGRI [en ligne http://www.momagri.org/FR/editos/Lettreouverte-a-Mariann-Fischer-Boel-Commissaire-europeenne-a-l-agriculture-et-au-developpementrural_129.html], consulté le 20/04/2012.

Ромво С (2008) La création d'entreprise pluriactive en milieu rural : vers un dispositif d'accompagnement par la Région Languedoc-Roussillon? Mémoire de Master 1 « Gestion des espaces ruraux, aménagement et développement local ». Université Paul Valéry, Montpellier.

ROBERTSON N, PERKINS H C, TAYLOR N (2008) Multiple job holding: interpreting economic, labour market and social change in rural communities. Sociologia Ruralis, 48 (4) : 331-350.

ROCHE B (2009) Le chômage continue d'augmenter au $2^{\text {ème }}$ trimestre 2009 en Languedoc-Roussillon comme en France.

In : INSEE Languedoc-Roussillon[en ligne

http://www.insee.fr/fr/regions/languedoc/default.asp?page=themes/conjoncture/conj0912/conj0912.htm], consulté le 10/04/2012.

SALEILLES S (2007) L'entrepreneur néo-rural et son réseau personnel : une étude exploratoire. Université Montpellier I, Montpellier.

SIMON A (2002) La pluriactivité dans l'agriculture des montagnes françaises. Un territoire, des hommes, une pratique. Presses Universitaires Blaise Pascal, collection Ceramac, Clermont Ferrand.

SOl ET CIVILISATION (2010) Gestion Territoriale des Emplois et des Compétences (GTEC), une démarche d'anticipation territoriale des activités et des compétences. Réseau Rural Français, Limoges.

TALLON H (2005) La pluriactivité dans le domaine de l'agriculture de l'environnement du tourisme et du patrimoine, source de dynamisme de l'activité et de l'emploi rural du Haut Languedoc. Thèse de Master of science "Développement rural et projets », CIHEAM/IAMM, Montpellier. 
TALlon, H, VAlETTE E, DéRIOZ P (2009) Les projets pluriactifs en zone rurale, entre ancrage territorial et mobilité spatiale ou professionnelle. Le cas des "hauts-cantons » du département de l'Hérault. XLVle Colloque de I'Association de Science Régionale De Langue Français. "Entre projets locaux de développement et globalisation de l'économie : quels équilibres pour les espaces régionaux", Clermont-Ferrand, France - Les 6, 7 et 8 juillet 2009.

TALLON H (2011a) Nous sommes les magiciens des petits revenus. Une analyse du statut du travail dans des projets de pluriactivité. In : BEGUIN P, DEDIEU B, SABOURIN E (éds.) Le travail en agriculture : son organisation et ses valeurs face à l'innovation. L'Harmattan, Paris.

TALLON H (2011b) Pluriactivité et accompagnement, un territoire à l'épreuve; une expérimentation par la formation menée dans le Haut Languedoc (Hérault, France). Thèse de doctorat de géographie. Université Paul Valéry, Montpellier.

THEVEnOt L (1996) Justification et compromis. In : CANTO-SPERBER M (dir) Dictionnaire d'éthique et de philosophie morale, PUF, Paris.

TRIBOULET P, LANGLET A (1999) Différenciation des espaces ruraux et insertion territoriale de l'agriculture. In INRA (Ed.), École-chercheur Économie spatiale et régionale, application à l'agriculture, l'agroalimentaire et l'espace rural. Le Croisic, 8, 9, 10 décembre 1999.

WEBER F (2009) Le travail à-côté. Une ethnographie des perceptions. EHESS, Paris. 